Result With this methodology of safety observation, the accident rate in terms of LTI (Lost Time Injury) and TRC (Total Recordable Cases) has been drastically brought down from 51 cases (2013-2014) to 23 cases (2016-2017) - 55\% reduction which includes 3 consecutive years of being LTI (Lost Time Injury) free.

Discussion Safety observation has proved an effective and excellent tool, which reinforces positive safety behaviour, raises safety awareness thereby motivating people to be committed for safety, corrects unsafe behaviours in a positive, proactive way leading to prevention of injuries and property losses.

\section{BEHAVIOURAL SAFETY - THE ULTIMATE STAGE IN ACCIDENT PREVENTION}

Una Feeney*. Department of Epidemiology and Public Health, College of Medicine and Health, National University of Ireland, Cork

\subsection{6/oemed-2018-ICOHabstracts.64}

Introduction To establish employee's perception of the safety culture in a multinational manufacturing facility, the status of safety management, in particular to measure the intangibles such as management commitment and employee beliefs and involvement in safety.

Method Cross sectional data was collected from 455 employees who voluntarily participated in the study. The research study was implemented based on a descriptive survey in order to estimate certain population parameters in relation to safety climate, safety culture and behavioural based safety in the workplace. They were asked to prioritise in relation to their perceived importance, the eight sections which had a total of fifty-four statements to address all of the important factors in safety management including beliefs and practices.

Results A valid sample of $89.2 \%$ was achieved for this study. The data showed a strong organisational commitment to safety, with 99\% employees agreeing with this statement. 90\% of employees agree that management commitment is evident; however, opportunities were identified in ensuring greater supervisory presence in the workplace. The lowest scoring values were the beliefs by employees that all accidents were preventable and that they were linked to personal behaviour. $65 \%$ agree that zero accidents are achievable, 23\% agreeing that if they had an accident it would be their own fault, these results remain a significant challenge for the organisation and may reflect a lack of understanding of the organisations injury data which reflects that over $50 \%$ of accidents are linked to behaviour.

Discussion This research study confirmed the validity of the one of the most important factors in the model of safety management, which is management commitment and employee involvement. If these 'soft' factors are identified and present within an organisation, this will then lead to excellence in safety.

\section{PSYCHOLOGICAL CONSEQUENCES OF ELECTRICAL ACCIDENTS AT WORK. INTERVIEWS WITH SWEDISH ELECTRICIANS}

\footnotetext{
${ }^{1,2}$ Sara Thomée*, ${ }^{1,2,3}$ Kristina Jakobsson. 'Occupational and Environmental Medicine, Sahlgrenska University Hospital, Gothenburg, Sweden; ${ }^{2}$ Department of Occupational and Environmental Medicine, Institute of Medicine, University of Gothenburg, Gothenburg, Sweden; ${ }^{3}$ Occupational and Environmental Medicine, Department of Laboratory Medicine Lund, Lund University, Lund, Sweden
}

10.1136/oemed-2018-ICOHabstracts.65
Introduction It is well known that electrical accidents can cause physical injury. Less well known is that long-term consequences may include emotional and cognitive problems. The objective was to explore electricians' experiences and perceptions of work-related electrical accidents, with focus on psychological short- and long-term consequences, including how contacts with health care services and the workplace had been perceived.

Methods Semi-structured interviews with 23 Swedish male electricians, aged 25-68, who had experienced at least one electrical accident and who had reported residual sensory, muscular, or mental symptoms. Data was analysed by means of qualitative content analysis, with the analysis keeping close to the areas of query and the electricians' statements.

Result Immediate emotional reactions included surprise, confusion, fear, anxiety, and anger, but also long-term consequences in terms of psychological dysfunction were seen. Experiencing a nolet-go situation was particularly stressful. The cause of the accident, and questions about guilt and blame, were central in the aftermath. Lack of knowledge and routine among health care professionals concerning electrical injury was reported, as well as lack of medical and psychological follow-up.

Discussion Long-term psychological consequences can be seen after occupational electrical accidents. Adequate handling at the workplace and from the health care services, including follow-up, could facilitate rehabilitation and return-to-work.

\section{KOREA'S RISK ASSESSMENT RECOGNITION SYSTEM AND PERFORMANCE}

HWANG Young-Kyu. KOSHA training institute Professor (KOREA Occupational Safety and Health Agency)

\subsection{6/oemed-2018-ICOHabstracts.66}

Introduction KOSHA has promoted risk assessment in the workplace so that employers can identify, evaluate, manage and improve hazardous factors in their workplaces. Risk assessment recognition is given to excellence sites that apply for risk assessment evaluation and are recognised as proper level in risk assessment from KOSHA. As a result, this system supports the establishment of a voluntary system

"Legal basis: Article 41-2 (Risk Assessment) of the KOREA ISHA and ACoP No. 2016-2017 of the Ministry of Labour Methods We carry out risk assessment recognition work as follows.

- Workplaces apply for risk assessment evaluation to KOSHA attaching risk assessment result and implementation rule

- KOSHA visit the work site within one month

- If there are no items that are less than 50 points out of 100 points and the total score is 70 points or more, those workplaces become candidates

- Accreditation committee composing of nine persons decides on risk assessment recognition

- Issuance of certificate within 5 days

Results The risk assessment recognition system has been implemented since 2013 and has achieved remarkable achievement by educating about 130000 employers, consulting 110000 places, recognising 14000 places, and reducing average industrial accident by $30.5 \%$ annually

Conclusion As the Korean government introduced the risk assessment recognition system to support self-safety management system 